\title{
Development of repellent strips for controlling the cigarette beetle, Lasioderma serricorne (Fabricius) (Coleoptera: Anobiidae)
}

\author{
Masatoshi HorI ${ }^{\dagger}$ \\ Leaf Tobacco Research Center, Japan Tobacco Inc.; Oyama, Tochigi 323-0808, Japan
}

(Received 1 September 2004; Accepted 21 February 2005)

\begin{abstract}
The residual activity of hinokitiol used as a repellent against the cigarette beetle was investigated. When hinokitiol was placed on filter paper at a dose of $1 \mathrm{mg} / \mathrm{cm}^{2}$, it exhibited remarkable residual activity $24 \mathrm{~h}$ after the treatment. However, no significant residual activity was observed after $48 \mathrm{~h}$ due to rapid sublimation $(90 \%$ of hinokitiol disappeared $48 \mathrm{~h}$ ). Therefore, the development of a device in the form of repellent strips designed to affect the slow release of hinokitiol was undertaken. The strips consisted of filter paper treated with hinokitiol, a polyethylene film bilayer in which the filter paper was sealed, and an aluminum sheet sandwiched between one side of the polyethylene strip and adhesive tape. Hinokitiol was slowly released from the repellent strips through the polyethylene film. The rate of hinokitiol release was inversely proportional to the thickness of the polyethylene film. Repellent strips attached around the slits of cardboard boxes containing a food-attractant lure prevented the beetles from entering the boxes. The number of beetles in the treatment boxes was less than $20 \%$ that observed in the control boxes $64 \mathrm{~d}$ after making the strips. The residual activity of the repellent strips was demonstrated to last up to a maximum of $92 \mathrm{~d}$ after they were produced.
\end{abstract}

Key words: Lasioderma serricorne; cigarette beetle; hinokitiol; repellent; residual activity

\section{INTRODUCTION}

The cigarette beetle, Lasioderma serricorne (Fabricius), damages a wide range of stored agricultural products, including cured tobacco leaves, cigarettes, cocoa beans, cereals, cereal products, oilseeds, pulses, spices, dried fruits and some animal products (Hill, 1990). This pest is the most serious insect threat to stored tobacco (Ryan, 1995), and is, at present, principally controlled by sanitization, remodeling equipment and fumigation. There are few control methods currently available that are capable of directly preventing the beetles from invading stored products.

The cigarette beetle is repelled by a variety of essential plant oils and their components such as shiso oil, savory oil, perillaldehyde and hinokitiol ( $\beta$-thujaplicine) (Hori, 2003, 2004a, b). Hinokitiol is particularly effective at repelling the beetles, even in the presence of tobacco odor, and has been demonstrated to prevent the beetles from both ap- proaching and settling (Hori, 2004b). Previous experiments have demonstrated that beetles were prevented from entering cardboard boxes containing cured tobacco leaves by the application of hinokitiol around the slits of the boxes (Hori, 2004c).

Practical application using a controlled release mechanism has proved difficult given that hinokitiol sublimes easily at room temperature (Nogami, 2001). Consequently, the development of a system that uses repellent strips to slowly release hinokitiol, yet strongly repel the cigarette beetle, was developed in this study.

\section{MATERIALS AND METHODS}

Insects. The adult cigarette beetles (4-8-d-old) used for the experimental runs were obtained from stock cultures that have been maintained for several years in the laboratory. They were reared on cured tobacco powder in plastic containers

\footnotetext{
${ }^{\dagger}$ Present address: Laboratory of Insect Science and Bioregulation, Graduate School of Agricultural Science, Tohoku University; Sendai 981-8555, Japan. E-mail: hori@bios.tohoku.ac.jp DOI: 10.1303/aez.2005.373
} 
(130 $\mathrm{mm}$ in diameter, $73 \mathrm{~mm}$ high) at $27 \pm 2^{\circ} \mathrm{C}$ and $70 \pm 5 \%$ relative humidity.

Hinokitiol. Hinokitiol (99.0\%) was obtained from Tokyo Kasei Kogyo Co., Ltd. (Tokyo, Japan).

Residual activity of hinokitiol. An eight-armed olfactometer was used for the assays. The olfactometer and methods used were essentially the same as those described in a previous paper (Hori, 2003). Fifty adult female beetles were released into the olfactometer connected to sample or control jars. The total number of beetles in the four sample tubes was compared with the number of beetles in the four control tubes $3 \mathrm{~h}$ after beginning each test. Filter paper (Advantec, No. 2, $10 \times 20 \mathrm{~mm}$ ), treated with a $10-\mathrm{ml}$ hinokitiol-acetone solution $(0.1 \mathrm{mg} /$ $\mu 1)$, was stored under fluorescent light $(550 \mathrm{~lx})$ at $25 \pm 1^{\circ} \mathrm{C}$ for periods of 1,24 or $48 \mathrm{~h}$ in a room $(532 \times 256 \mathrm{~cm})$ maintained at a constant environment. The paper samples thus prepared were subsequently placed into the sample jar of the olfactometer. As a control, filter paper treated with acetone was subjected to the same exposure treatments. These paper samples were placed in the control jar. Each test was replicated four times under light conditions $(550 \mathrm{~lx})$ at $25 \pm 1^{\circ} \mathrm{C}$. The data from the four replicates were pooled and transformed into an excess proportion index $(E P I)$ according to the following formula:

$$
\begin{aligned}
& E P I=(n s-n c) /(n s+n c)=2 P S-1 \\
& P S=n s /(n s+n c)
\end{aligned}
$$

where $n s$ and $n c$ represent the total number of beetles in the sample and control tubes, respectively, and $P S$ represents the proportion of beetles in the sample tubes (Sakuma and Fukami, 1985). The $95 \%$ fiducial limits for $P S$ were calculated from the critical values of the variance ratio $F$ and then transformed into $E P I$ values.

Sublimability of hinokitiol. A sheet of filter paper (Advantec, No. 2, $100 \times 100 \mathrm{~mm}$ ), treated with a hinokitiol-acetone solution (hinokitiol: $1 \mathrm{mg} /$ $\left.\mathrm{cm}^{2}\right)$, was exposed to light $(550 \mathrm{~lx})$ at $25 \pm 1^{\circ} \mathrm{C}$ in a constant environment room. The sheet was weighed at each of the sample times, and sublimation and the residual amount of hinokitiol were calculated by weight loss from the sheet. Ten such replications were performed.

Design of repellent strips. Repellent strips consisted of filter paper treated with hinokitiol at a dose of $10 \mathrm{mg} / \mathrm{cm}^{2}$; a polyethylene outer covering in which the filter paper was sealed, and an aluminum strip sandwiched between the polyethylene cover and adhesive tape (Fig. 1). The sheet of filter paper (Advantec, No. 2, $20 \times 25 \mathrm{~cm}$ ) was uniformly covered with hinokitiol $(5 \mathrm{~g})$ before being heated in an oven (Windy Oven WFO-600SD, Tokyo Rikakikai Co., Ltd., Tokyo, Japan) at $60^{\circ} \mathrm{C}$ for $7 \mathrm{~min}$, causing the hinokitiol to melt and be absorbed by the filter paper. The hinokitiol in the paper sheets was then allowed to solidify by exposure to room temperature $\left(25 \pm 1^{\circ} \mathrm{C}\right)$ for $8 \mathrm{~min}$ before being cut into strips $(1 \times 25 \mathrm{~cm})$ that were then sandwiched between two polyethylene sheets (thickness: 30,50 or $100 \mu \mathrm{m}$; WDHAEC43, Sanplatec Co., Ltd., Osaka, Japan). These two polyethylene sheets were heat-sealed together along the limb of the hinokitiol-saturated paper strip. An aluminum sheet was then sandwiched between the polyethylene strip and a strip of adhesive tape. The hinokitiol was thus released only from the one side of the polyethylene film of the repellent strips (Fig. 1).

Release rate of hinokitiol from repellent strips. Repellent strips made using 30-, 50- or 100$\mu \mathrm{m}$ polyethylene film were left in the constant environment room at $25 \pm 1^{\circ} \mathrm{C}$ under a photoperiod regime of $14 \mathrm{~L}: 10 \mathrm{D}$. The repellent strips were weighed on each of the sample days, and the sublimated and residual amounts of hinokitiol were calculated by weight loss of the strips. Repellent strips not sealed in polyethylene film were used as a control. Four replications were performed for each thickness of polyethylene film.

Evaluation of the practicability of repellent strips in a quasi-field test. Cigarette beetles were released into a room and allowed to choose between the food-attractant lures contained in the treatment and control cardboard boxes. The room used for the experiment, and the location of the treatment and control boxes in the room were the same as those described in a previous study (Hori, $2004 \mathrm{c})$. Each box $(27 \times 37 \times 15.6 \mathrm{~cm})$ had a slit measuring $1 \times 25 \mathrm{~cm}$ facing the roof to allow the beetles to enter the box. Repellent strips with 100$\mu \mathrm{m}$ polyethylene film were attached around the slit of the treatment box. In the control, dummy strips without hinokitiol were attached in the same way as the treatment box. A food-attractant lure $(14 \times 3.3 \mathrm{~mm}$ high) for the cigarette beetle (New Serrico $^{\circledR}$, Fuji Flavor Co., Ltd., Tokyo, Japan) was 


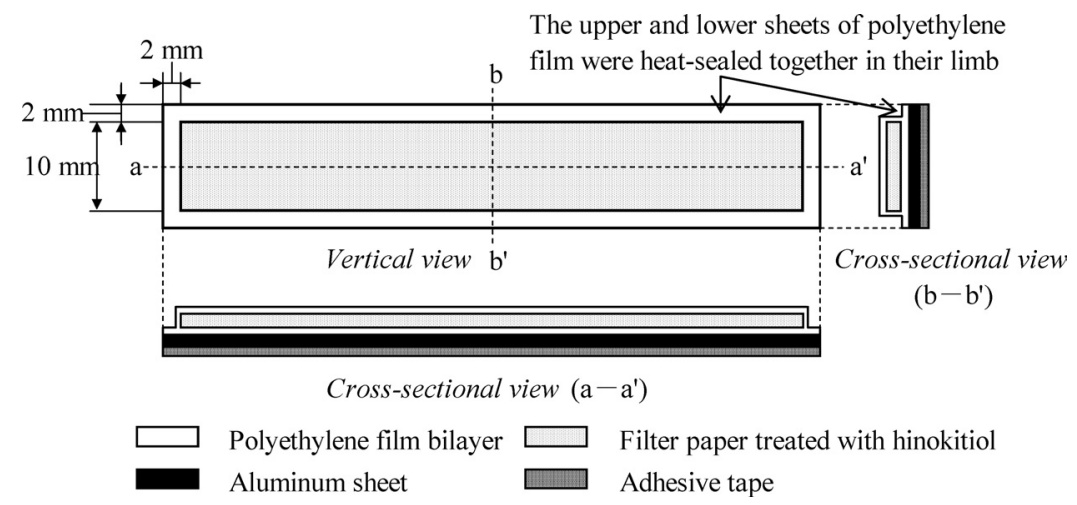

Fig. 1. Constitution of the repellent strips. Repellent strips consisted of the filter paper treated with hinokitiol; a polyethylene film bilayer in which the filter paper was sealed, and an aluminum sheet sandwiched between one of the polyethylene surfaces and adhesive tape.

placed at the bottom of the cardboard box. Threethousand adult beetles were released at an equidistant position from each box. After $40 \mathrm{~h}$, each box was dismantled and the number of beetles in each box was counted. The repellent and dummy strips attached to the boxes were collected after each trial and incubated in a room held at a constant environment, $25 \pm 1^{\circ} \mathrm{C}$, and under a photoperiod regime of $14 \mathrm{~L}: 10 \mathrm{D}$ until the time of the next trial. The sum of the number of the beetles in the two treatment boxes was compared with the number found in the two control boxes. Four replications were performed for each test. The location of the control and treatment boxes was alternated for each replicate. The tests were conducted under dark conditions at $25 \pm 1^{\circ} \mathrm{C}$. The data of the four replications were pooled and transformed into EPI values using the formula given above.

\section{RESULTS AND DISCUSSION}

\section{Residual activity and sublimability of hinokitiol}

The repellency of hinokitiol against cigarette beetles was strong at 1 and $24 \mathrm{~h}$ after application to the filter paper: EPI values were approximately -1.0 and -0.8 at 1 and $24 \mathrm{~h}$ after application of the hinokitiol, respectively (Fig. 2). However, repellency was not apparent after $48 \mathrm{~h}$. Hinokitiol sublimed rapidly on the filter paper, with approximately 70 and $90 \%$ sublimation achieved at 24 and $48 \mathrm{~h}$ after initial application, respectively. Essentially all hinokitiol was sublimed at $72 \mathrm{~h}$ (Fig. 3). The sublimation of hinokitiol $48 \mathrm{~h}$ after application was approximately $10 \%$ of the amount at $1 \mathrm{~h}$ after application.

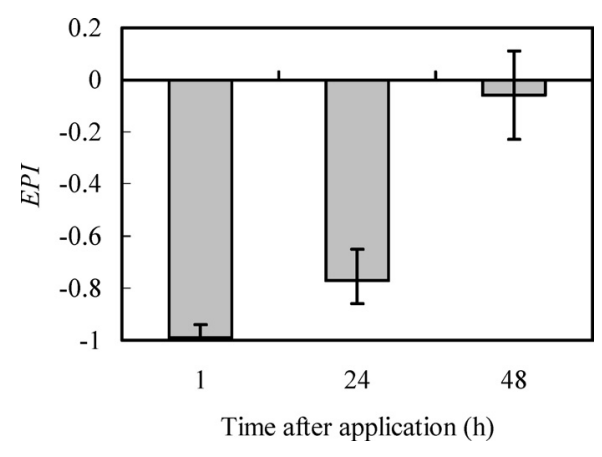

Fig. 2. Residual activity of hinokitiol applied to filter paper. A piece of filter paper treated with hinokitiol was exposed for 1,24 , or $48 \mathrm{~h}$ in a room before testing the repellency of the treated paper each time against the cigarette beetles using an olfactometer: $E P I=(n s-n c) /(n s+n c) ; n s, n c$ : total number of $L$. serricorne in the sample and control tubes, respectively. Error bars indicate 95\% fiducial limits of EPI.

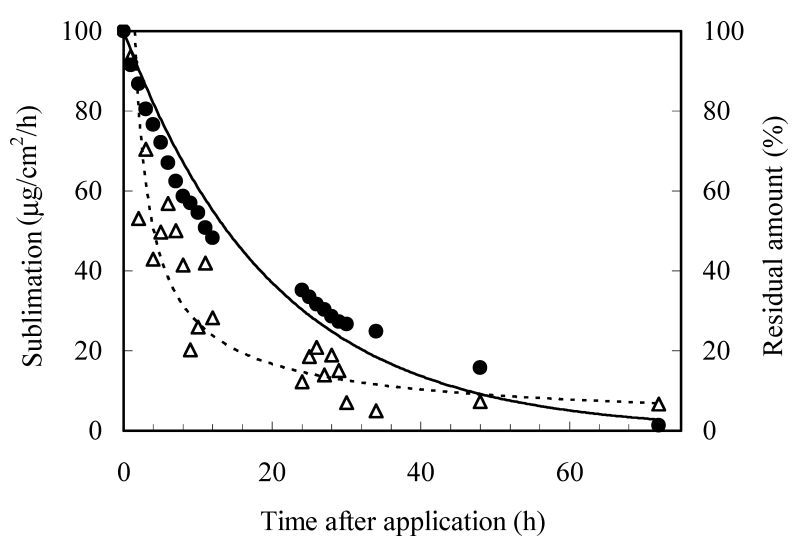

Fig. 3. Sublimability of hinokitiol applied to filter paper. A sheet of filter paper treated with hinokitiol and exposed in a room maintained at a constant environment. Sublimation and residual hinokitiol were calculated by weight loss of the sheet: Sublimation of hinokitiol from filter paper $(\triangle)$; Residual amount of hinokitiol in filter paper $(\mathbf{O})$. 

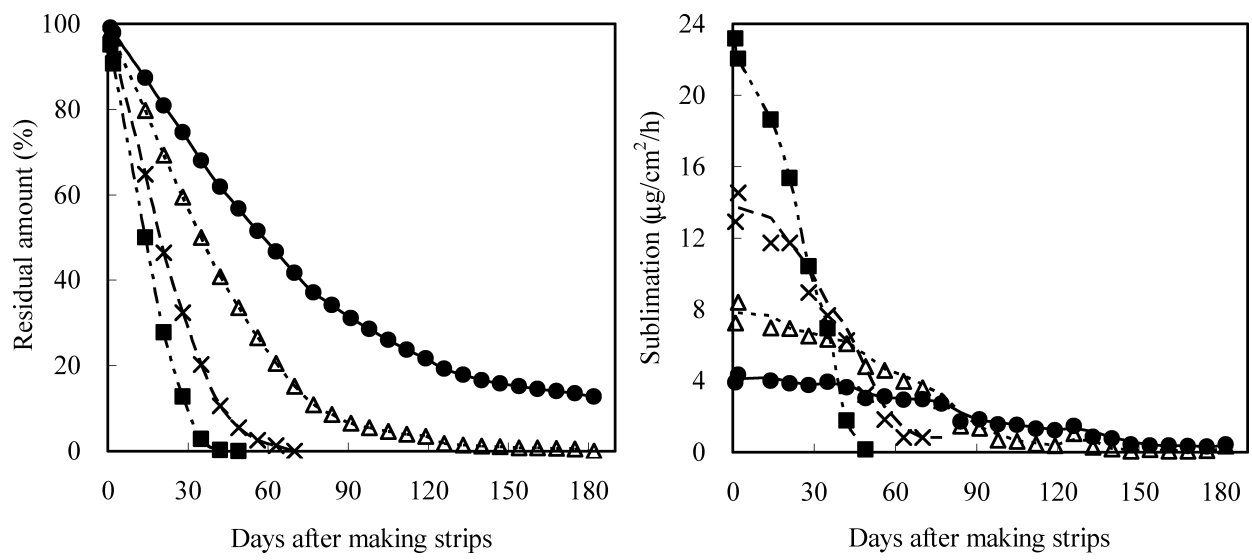

Fig. 4. Release rate of hinokitiol from each repellent strip. Repellent strips with 30-, 50- or 100- $\mu$ m polyethylene film were exposed in a room maintained at a constant environment, with sublimation and residual amount of hinokitiol calculated by weight loss from the strips: Repellent strips without polyethylene film (Control strips) (ם); Repellent strips with 30- $\mu$ m polyethylene film $(\times)$; Repellent strips with 50- $\mu$ m polyethylene film $(\triangle)$; Repellent strips with $100-\mu \mathrm{m}$ polyethylene film $(\mathbf{O})$.

\section{Practicability of repellent strips}

Hinokitiol sublimed rapidly from the control strips that were not sealed in polyethylene film (Fig. 4). Conversely, the rate of hinokitiol release decreased as the thickness of the polyethylene film increased. Repellent strips with 50 or $100-\mu \mathrm{m}$ polyethylene film released hinokitiol at a stable rate for more than 2 mo. The repellent strips strongly prevented the beetles from entering the cardboard boxes containing the food-attractant lure for up to 3 mo (Fig. 5). The number of beetles entering the experimental boxes was less than $20 \%$ of that observed in the control boxes after 2 mo of making the strips $(E P I=$ ca. -0.7$)$, and approximately $35 \%$ of the control boxes 1 mo later $(E P I=$ ca. -0.5$)$. Residual activity was not observed at $120 \mathrm{~d}$ after making the strips.

Residual activity is a very important issue regarding the practical application of insecticides, repellents, and attractants. Although hinokitiol has a strong repellency against the cigarette beetle, it sublimes rapidly at room temperature. Therefore, controlled release of hinokitiol is necessary in order to use the repellent practically. Various types of controlled release techniques, such as plastic capsules, rubber caps and various other mechanisms, have been employed with pheromone devices (Nakamura and Tamaki, 1983). In this study, polyethylene film was used to affect the controlled release of hinokitiol. The initial release rate of hinokitiol from the repellent strips with $100-\mu \mathrm{m}$ polyethylene film was approximately $3.9 \mu \mathrm{g} / \mathrm{cm}^{2} / \mathrm{h}$

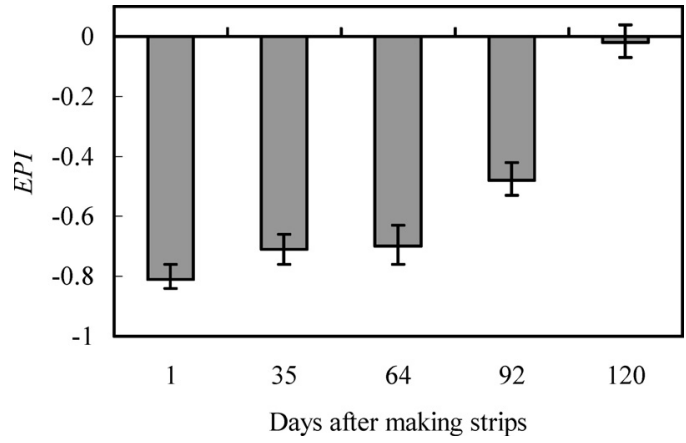

Fig. 5. Repellency of the repellent strips against Lasioderma serricorne in a quasi-field test. The beetles were released into the room and were allowed to choose between the treatment and control boxes containing food-attractant lures. The experimental and control strips were attached around the slits of the treatment and control boxes. Both strips were collected from boxes after each trial, and were left in the constant-environment room until the next trial: $E P I=(n s-n c)$ / $(n s+n c) ; n s, n c$ : sum of the number of L. serricorne in the two treatment and two control boxes, respectively. Error bars indicate $95 \%$ fiducial limits of EPI.

and less than $20 \%$ that observed in the control strips. Even after $3 \mathrm{mo}$, the release rate of hinokitiol was $1.8 \mu \mathrm{g} / \mathrm{cm}^{2} / \mathrm{h}$ and the beetles were significantly repelled. The practical application of insect repellents for such purposes requires that residual activity be maintained for at least 1 mo. The repellent strips developed in this study thus appear to meet the requirements of a slow-release vehicle for insect repellent.

The beetles avoided approaching and walking on the filter paper treated with hinokitiol (Hori, 
$2004 b, c)$. When the repellent strips were attached around the slits of the cardboard boxes, the beetles would not approach the slits. In instances where the beetles were observed to approach the repellent strips, they would avoid contact with them and leave immediately. Consequently, the beetles were prevented from entering the cardboard boxes.

Hinokitiol is corrosive on iron (Nogami, 2001). The repellent strips developed in this study employed a layer of aluminum sheeting on the side used for adhesion, through which the repellent could not penetrate. Consequently, when attached to an iron plate, the strips were not observed to cause corrosion (Hori, unpublished). Hinokitiol is a photolytic chemical (Nogami, 2001) and it was observed that the hinokitiol-impregnated filter paper of the repellent strips gradually photolyzed and became yellow over time. However, because of its residual activity $(2 \mathrm{mo})$, the photolysis of hinokitiol is unlikely to negatively impact the potential for practical application of the repellent strips.

Cigarette beetles have the propensity to enter, and are commonly found in narrow openings. The beetles can easily invade the packaging of stored products through extremely narrow openings in the packages. Furthermore, curing sheds, storage facilities and food processing plants have many narrow gaps. The beetles are able to enter these gaps easily. Once inside they stay and multiply before moving on to other openings or stored products. Attaching the repellent strips around possible access points to these materials or places may thus reduce the risk associated with product losses and claims as their access to these narrow gaps is inhibited.

\section{ACKNOWLEDGEMENTS}

I wish to thank Prof. K. Matsuda, Tohoku University for kindly reviewing the manuscript.

\section{REFERENCES}

Hill, D. S. (1990) Pests of Stored Products and Their Control. Belhaven Press, London. 274 pp.

Hori, M. (2003) Repellency of essential oils against the cigarette beetle, Lasioderma serricorne (Fabricius) (Coleoptera: Anobiidae). Appl. Entomol. Zool. 38: 467473.

Hori, M. (2004a) Repellency of shiso oil components against the cigarette beetle, Lasioderma serricorne (Fabricius) (Coleoptera: Anobiidae). Appl. Entomol. Zool. 39: 357362.

Hori, M. (2004b) Repellency of hinokitiol against the cigarette beetle, Lasioderma serricorne (Fabricius) (Coleoptera: Anobiidae). Appl. Entomol. Zool. 39: 521526.

Hori, M. (2004c) Evaluation of the practicability of hinokitiol as a repellent against the cigarette beetle, Lasioderma serricorne (Fabricius) (Coleoptera: Anobiidae). Appl. Entomol. Zool. 39: 699-704.

Nakamura, K. and Y. Tamaki (1983) Monitoring of insect pests with sex pheromone.-1. Source of attraction and trap-. In Sex Pheromone and Insect Pest Control-Experiment and Utility -. Kokon Publishing Co., Tokyo, pp. 54-65 (in Japanese).

Nogami, M. (2001) New antibacterial inorganic materials hybridized with hinokitiol. Bio Industry 18: 35-40 (in Japanese).

Ryan, L. (1995) Post-Harvest Tobacco Infestation Control (L. Ryan ed.). Chapman \& Hall, London. 155 pp.

Sakuma, M. and H. Fukami (1985) The linear track olfactometer: An assay device for taxes of the German cockroach, Blattella germanica (L.) (Dictyoptera: Blattellidae) toward their aggregation pheromone. Appl. Entomol. Zool. 20: 387-402. 